\title{
APPROACHES TO DISTINGUISH 'REAL' CHANGES FROM 'UNREAL' CHANGES BASED ON MULTI-TEMPORAL 2D BUILDING FOOTPRINT DATA
}

\author{
M. Schorcht ${ }^{1, *}$, R. Hecht ${ }^{1}$, G. Meinel ${ }^{1}$ \\ ${ }^{1}$ Leibniz Institute of Ecological Urban and Regional Development, Dresden, Germany - (m.schorcht, r.hecht, g.meinel)@ioer.de
}

\author{
Commission IV, WG IV/3
}

KEY WORDS: building stock, data correction, change detection, 2D cadastre building data, European Settlement Map (ESM)

\begin{abstract}
:
Building footprint data from National Mapping and Cadastral Agencies are available in Germany for 7 years as a uniform, nationwide geospatial data set and are updated annually. These multi-temporal building data sets can form the basis for the application of change detection techniques to derive national figures on dynamics in the building stock. Since these building data sets have only been built up in recent years, it is necessary to distinguish real changes from false changes. This is done by applying vector geometry-based operations and statistical analyses, which are presented in this article. Furthermore, by the additional use of the raster dataset Copernicus - European Settlement Map (classified, resolution $2.5 \mathrm{~m}$ ) it is approximately possible to estimate whether it is a correct change or not. The advantage of this approach is that large-scale comparable results can be derived simply and quickly based on uniform basic data.
\end{abstract}

\section{INTRODUCTION}

Small-scale land use changes on the level of individual buildings are playing an increasingly important role in urban and regional planning and environmental sciences. Changes in the building stock are used, for example, for material flow analyses and modelling (Tanikawa \& Hashimoto, 2009, Kleemann et al., 2017) or for demolition analyses (Aksözen et al., 2017). Furthermore, by analysing the degree of building coverage over time, it is possible to quantify densification processes in inner and outer urban areas (Jehling et al., 2016).

For some years now, National Mapping and Cadastral Agencies offer vector data sets containing the building footprint information with nation-wider coverage. These datasets are regularly updated and offer the opportunity for tracking changes over time (Hartmann et al., 2016). However, since the building datasets of recent years were still under construction, the detected changes are a composition of "real" and "unreal" changes. The "unreal" changes are incorrectly and thus false positives. Therefore, the aim of this study is to develop a robust approach for change detection in multi-temporal 2D building data sets. The change detection approach should be able to recognize the evolution of buildings over time. According to the typology of spatio-temporal processes proposed by Claramunt and Thériault (1996) the evolution of a single entity can be a basic change (appearance, disappearance), a transformation (expansion, contraction, deformation) as well as a movement (displacement, rotation), the latter being rarely applicable to buildings.

This paper presents solutions to distinguish between the real changes and false changes considering effects due to various reasons, such as transformation errors, duplicates, or small inaccuracies of the 2D geometry. Most of the false changes can be detected applying vector-based operations. Missing and subsequent additions of buildings (e.g. entire building categories were added at a later point in time) may be indicated by a statistical analysis. Finally, we show that subsequent additions can be detected by using the European Settlement Map (ESM, classified raster layer with a resolution of $2.5 \mathrm{~m}$ ) as an additional data source.

\section{USED BASIC DATA}

\subsection{Official Building Polygons of Germany (HU-DE)}

As input we use is a Germany-wide and multi-temporal building data set called Amtliche Hausumringe Deutschland (HU-DE) that contains all building footprints from the cadastral agencies produced by the federal surveying and mapping authorities of the German States (6 time slices, from 2011 to 2016). These have been produced according to uniform criteria and therefore have the same geometric accuracy (in the centimeter range). The data set is collected and distributed by the Central Office for House Coordinates, Building Polygons and 3D Building Models (ZSHH, 2018). The 2016 data set contains more than 53 million objects.

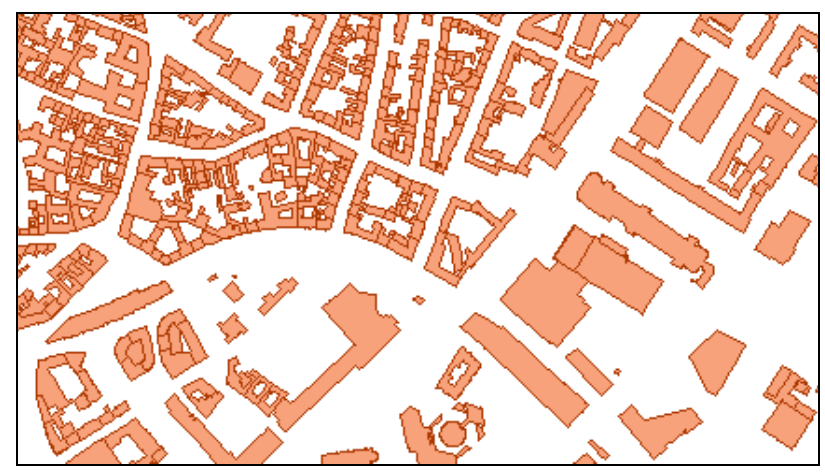

Figure 1. Example of building polygons (BKG 2018) 
As can be seen in Figure 1, the polygons do not always correspond to a building, so that no statement can be made about the number of buildings without a pre-processing. For this study, only the change in the building area is investigated.

Figure 2 shows the total sum of building area from 2011 to 2016. It shows unusual increases in the building area between 2015 and 2016 for Lower Saxony and Baden-Württemberg. As this product is relatively new, adjustments, additions and corrections have been made over the years that do not correspond to any real changes. Such adjustments were apparently also carried out by the federal states, which are leading to such effects.

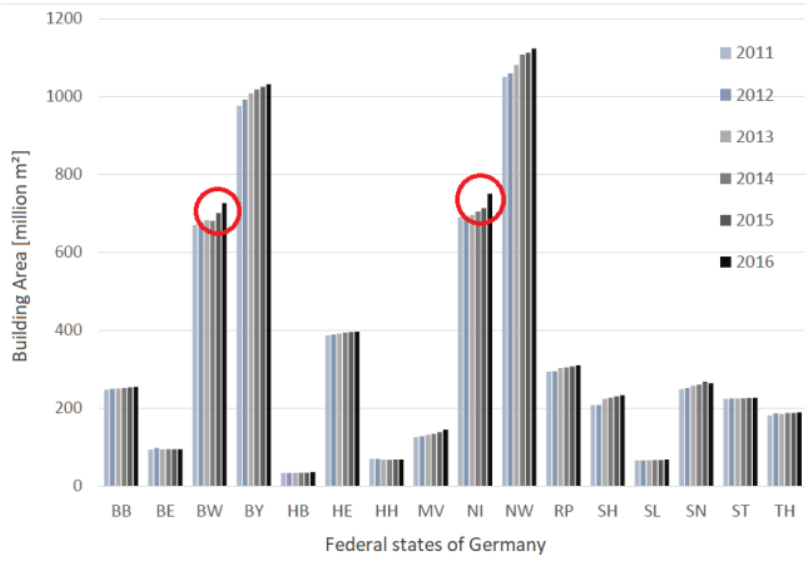

Figure 2. Total area of the building polygons of all 16 German federal states from 2011 to 2016 (unusual increases are marked with a red circle)

\subsection{Copernicus Land Monitoring Service - European Settlement Map 2012 (Release 2017)}

The European Settlement Map 2012 - Release 2017 is a classified spatial raster dataset mapping human settlements in Europe with a spatial resolution of $2.5 \mathrm{~m}, 10 \mathrm{~m}$, and $100 \mathrm{~m}$ (Florczyk et al., 2016).

It is based on SPOT5 and SPOT6 satellite imagery and has been produced with GHSL technology (and if available the use of OSM streets and buildings) by the European Commission, Joint Research Centre, Institute for the Protection and Security of the Citizen, Global Security and Crisis Management Unit (Copernicus, 2017).

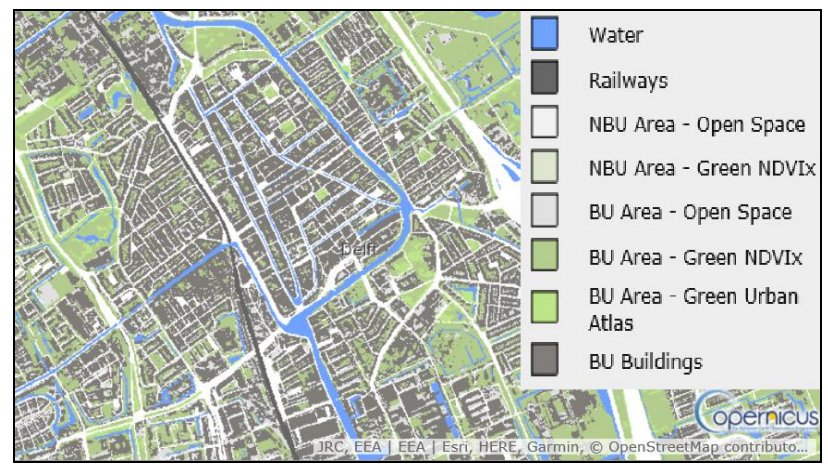

Figure 3. Example of European Settlement Map 2012 - Release 2017 (Copernicus, 2017)

\section{DETECTION AND CORRECTION OF CHANGES}

The causes of incorrectly detected changes can be very different. The most important sources of errors and possible solutions for their automatic detection and correction are presented here. The correction can be either applied on the input layers or the difference layer as a result of a geometric intersection of the two input layers.

\subsection{Errors due to elimination of duplicates and overlaps}

Problem: Older datasets sometimes contain duplicate (or partly overlapping) polygons, which in case of a correction (on side of producer) lead to an apparent decrease of the building area.

Approach for correction: With identical geometry, these duplicates can be removed easily with standard operations available in GIS Software (e.g. ArcGIS: Find Identical, FME: Matcher). However, these duplicates do not always have an identical geometry or are just partly overlapping polygons. In these cases a dissolve of both polygons is performed. Duplicates can be a big source of errors, occurs systematically and scattered. It is crucial that the duplicates are removed before the intersection, since they are no longer noticeable in the difference layer.

\subsection{Errors due to changes of the geometry}

\subsubsection{Geometric representation (sliver polygons):}

Problem: False changes are caused by small geometric deviations due to changing data basis or changing algorithms in the manufacturing process (Champion et al., 2010).

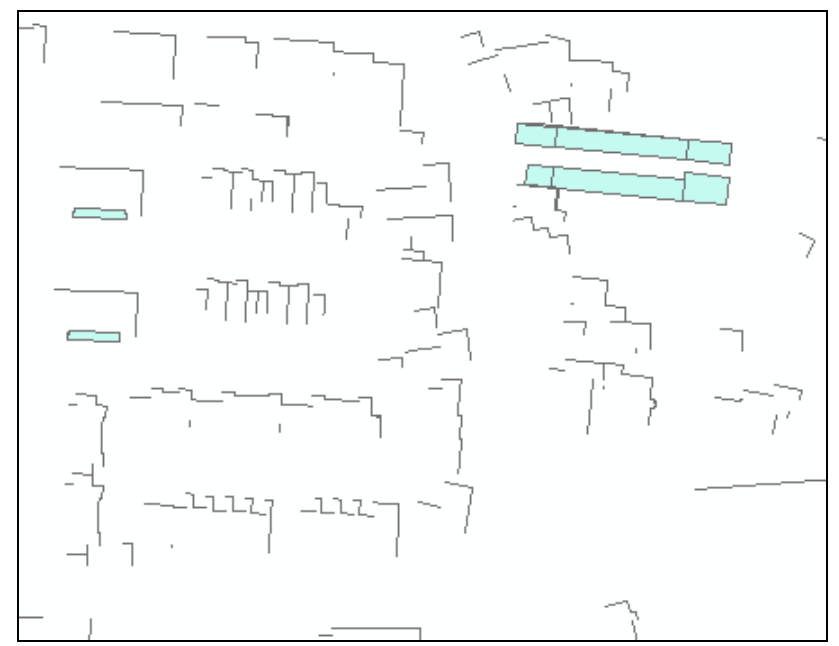

Figure 4. Difference-Layer with sliver polygons

This error can lead to incorrect changes in both directions (added or removed polygon parts), especially if the error is greater than the actual change.

Ideally, these differences cancel each other out (if added areas are equal to removed areas), but often there is a one-sided change that leads to a general over- or under-estimation (see Figure 4). The errors caused by this can be greater than the real change itself and must therefore be corrected.

Approach for correction: For smaller deviations $(<0.5 \mathrm{~m})$ it is sufficient to reduce the geometrical tolerance of the datasets. A greater tolerance than 0.5 leads to unwanted deformations of the 
objects, which in turn falsifies the statement. For the removal of wider sliver polygons, the method of morphological filtering known from image processing is suitable. By the use of the opening operator (erosion and dilation) these sliver polygons can be removed. As the corners of the objects round off, the corrected opening (Schorcht et al., 2016) is used, which avoids this effect (see Figure 5).

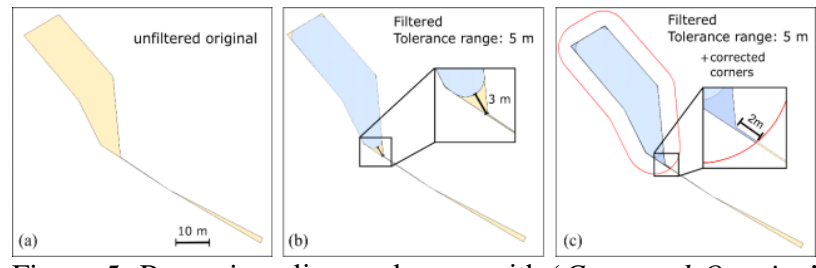

Figure 5. Removing sliver polygons with 'Corrected Opening' (Schorcht et al., 2016)

Formally, the corrected opening can be stated as follows:

$$
A_{C}=\left(\left(A \circ B_{1}\right) \oplus B_{2}\right) \cap A
$$

where $\quad \mathrm{A}_{\mathrm{c}}=$ corrected object

$\mathrm{A}=$ original object

$\mathrm{B}_{1}=$ structure element (shift tolerance)

$\mathrm{B}_{2}=$ structure element (corner correction)

$\circ=$ opening

$\oplus=$ dilation

$\cap=$ intersection

With the corrected opening real building extensions (true change) can also be distinguished from unwanted narrow deviations (false change), because all object changes wider than the tolerance range (here $2.5 \mathrm{~m}$ ) remain unfiltered.

\subsubsection{Positional shifts:}

Problem: Another problem in detecting changes can occur if date transformations have been performed (e.g. from DHDN_Gauss_Krueger to UTM_ETRS89) that lead to small offsets in the difference layer (sliver polygons up to $2.5 \mathrm{~m}$ ). Although these offsets are not relevant when considering the total building changing area (because the size of the added area must be the same as the removed one), it plays a only a role if one wants to investigate further separate analyses on added and removed areas.

Approach for correction: Therefore, these small offsets are also removed using morphological filtering (see above).

\subsection{Errors due to subsequent additions in the data set}

Problem: Adding missing or removing unintended objects at a later state (e.g. smaller objects such as garages have been added later on a large scale in Germany) leads to extensive changes in the dataset. The influence of these amendments can be so great that the municipal boundary becomes apparent in the differential layer (Figure 6).

Approach 1 for correction: Subsequent additions can partly be recognized by statistical analyses. For this purpose, size classes are formed at the municipal level and checked to see whether they change conspicuously. However, this approach can only identify large subsequent additions. If the proportion of a size classe changes by more than $10 \%$ per year, a subsequent addition is very likely, or there was a change in the modelling of building parts.

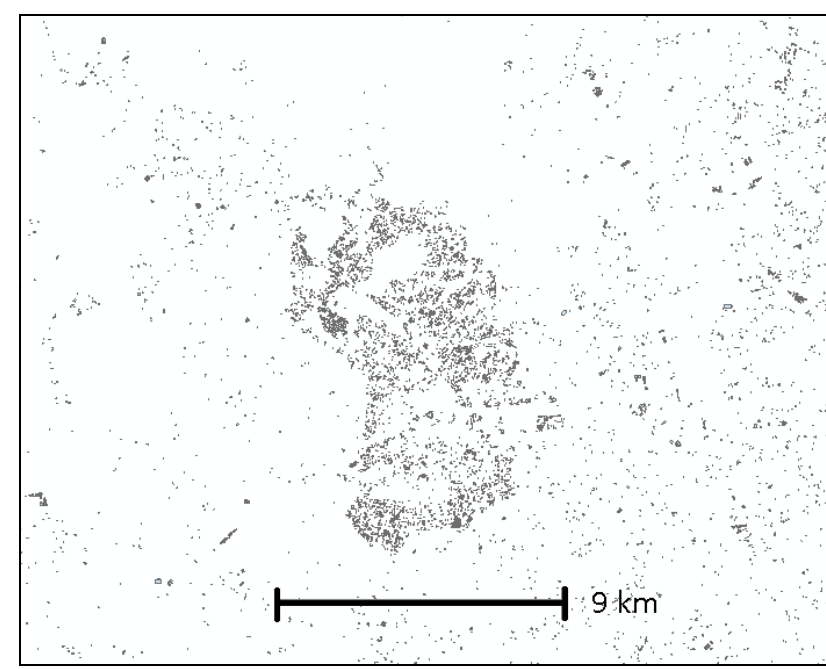

Figure 6. Difference-Layer with afterwards added small objects

Approach 2 for correction: Another approach to identify incorrect changes is to use the ESM as an additional data source. For this, the supposedly new building polygons (change from 2012 to 2016) are overlaid with the ESM (from 2012) and the number of building pixels within the polygon is analyzed (see Figure 7). If more than $80 \%$ of the polygon is filled with building pixels, it is assumed to be an addendum to be excluded from the difference layer. Initial research has shown that most of the false changes can be detected and corrected using this approach. However, further analyses are necessary.

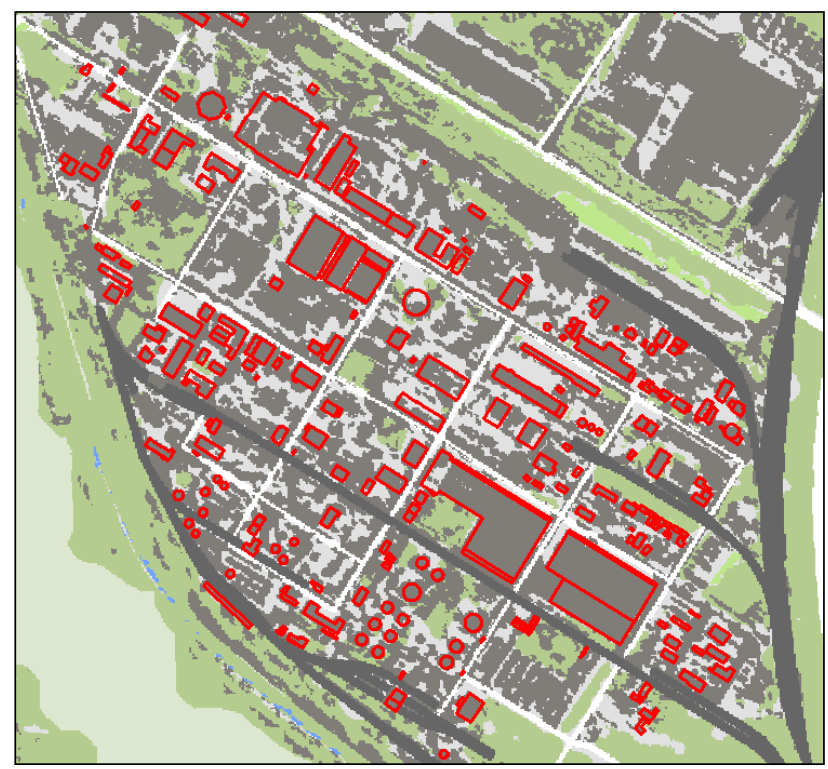

Figure 7. Combination of ESM with afterwards added building polygons; background: ESM 2012; red outlined polygons: false building change (2012-2016)

\section{RESULTS}

The approaches were tested on the data set of the federal state of North Rhine-Westphalia with the exception of approach 2 (combination with ESM, see 3.3), which has so far only been tested on a smaller study area. 
Table 1 shows the results of the size class analysis. It can be clearly seen that the proportion of small objects (area smaller than $15 \mathrm{~m}^{2}$ ) has been significantly increased. These are mainly objects that have been added later. Buildings under $40 \mathrm{~m}^{2}$ were therefore excluded from the change analysis, as they would otherwise have a significant influence on the results.

\begin{tabular}{|l|c|c|c|}
\hline Class & Area size $\left[\mathrm{m}^{2}\right]$ & \multicolumn{2}{|c|}{ Proportion [\%] } \\
\hline & & 2012 & 2016 \\
0 & $0-10$ & 0.1 & 0.4 \\
1 & $10-15$ & 0.3 & 0.6 \\
2 & $15-20$ & 1.6 & 1.8 \\
3 & $20-25$ & 1.3 & 1.4 \\
4 & $25-30$ & 1.2 & 1.3 \\
5 & $30-35$ & 1.2 & 1.2 \\
6 & $35-40$ & 1.2 & 1.2 \\
7 & $40-45$ & 1.1 & 1.1 \\
8 & $>45$ & 92.0 & 91.1 \\
\hline
\end{tabular}

Table 1. Share of the area classes compared from 2012 to 2016 in North-Rhine-Westphalia

Table 2 shows the results of the change analysis for the whole of North Rhine-Westphalia, taking into account the exclusion of small area classes. Overall, nearly a quarter $(23.9 \%)$ of all changes are attributed to such small objects.

\begin{tabular}{|l|c|c|}
\hline Excluded area & \multicolumn{2}{|c|}{ Change } \\
\hline & million $\mathrm{m}^{2}$ & $\%$ \\
None (original) & 71 & 100.0 \\
$<10 \mathrm{~m}^{2}$ & 67 & 94.4 \\
$<20 \mathrm{~m}^{2}$ & 61 & 85.9 \\
$<40 \mathrm{~m}^{2}$ & 54 & 76.1 \\
\hline
\end{tabular}

Table 2. Effect of small objects in North-Rhine-Westphalia (2012 to 2016)

The effects of sliver polygons are not as serious as the subsequent additions. Overall, $15 \%$ of the changes in NorthRhine-Westphalia could be attributed to such small spatial offsets. However, these effects are more unevenly distributed. If a community is affected heavily, extreme deviation may occur, as Table 3 illustrates.

\begin{tabular}{|l|c|c|}
\hline Sliver-tolerance & \multicolumn{2}{|c|}{ Change } \\
\hline $\mathrm{m}$ & ha & $\%$ \\
0.0 (original) & 240.7 & 100 \\
1.0 & 169.4 & 70.4 \\
2.5 & 112.6 & 46.8 \\
5.0 & 76.9 & 32.0 \\
\hline
\end{tabular}

Table 3. Effect of sliver polygons in one heavily affected municipality (2012 to 2016)

If the difference layers are used for further analyses, it is generally recommended to remove these sliver polygons. In most cases these small geometrical deviations cancel each other out, but a separate view of de- and increases can lead to extremely overestimated values.

Based on initial research, it can be roughly estimated that by additional using ESM about $90 \%$ of the remaining false changes are detected, but about $20 \%$ of the true changes are also excluded.

\section{CONCLUSION AND OUTLOOK}

In this paper an overview of different approaches to distinguish real from unreal changes based in multi-temporal $2 \mathrm{D}$ data sets has been given. It was shown how duplicates, overlays and positional inaccuracies of the building data sets can be detected and eliminated using geometric operations. In addition, an approach of size class analysis was presented, with which more extensive subsequent added buildings, which do not correspond to any real changes, can be recognized.

Since especially the buildings added afterwards represent a major source of errors in change detection, the European Settlement Map (classified, $2.5 \mathrm{~m}$ ) was used for an additional consistency check. This step is still under development and further testing is needed.

The approaches have so far only been tested on small test areas and still lead to false positives. It could be shown that the ESM layer has great potential to support the detection process in the future.

There are other, still unconsidered errors that can lead to false changes. For example, there are known changes in the modelling of building parts, which currently can also result in unreal changes. However, an unknown error is always retained, since not all real object changes are contained in the building datasets and these can not be derived with the given data basis.

In the future, processing results of the other federal states may provide further information on the suitability of the process and the quality of official building footprint data.

\section{ACKNOWLEDGEMENTS}

We would like to thank the Federal Ministry for Economic Affairs and Energy (BMWi) for funding the project ENOB:dataNWG (FKZ 03ET1315).

\section{REFERENCES}

Aksözen, M., Hassler, U., Rivallain, M., and Kohler, N., 2017. Mortality analysis of an urban building stock. In: Building Research \& Information, 45:3, pp. 259-277, https://dx.doi.org/10.1080/09613218.2016.1152531.

Claramunt, C., and Thériault, M., 1996. Toward semantics for modelling spatio-temporal processes within GIS. In: Advances in GIS Research II, pp. 47-63. Kraak, M. J., and Molenaar, M. (Eds.), Taylor and Francis, London.

BKG, 2018. Bundesamt für Kartographie und Geodäsie (BKG), Sonstige Produkte - amtliche Hausumringe Deutschland (HUDE). https://www.bkg.bund.de/DE/Produkte-undServices/Shop-und-Downloads/DigitaleGeodaten/SonstigeProdukte/SonstigeProdukte.html.

Champion, N, Boldo, D., Pierrot-Deseilligny, M., and Stamon, G., 2010. 2D building change detection from high resolution satellite imagery: A two-step hierarchical method based on 3D invariant primitives. French Mapping Agency, MATIS Lab., 2-4 Avenue Pasteur, 94160 Saint Mandé, Fance https://doi.org/10.1016/j.patrec.2009.10.012. 
Copernicus, 2017. Land Monitoring Service, European Settlement Map (ESM) - Release 2017. European Commission, Joint Research Centre, Institute for Protection and Security of the Citizen

https://land.copernicus.eu/pan-european/GHSL/europeansettlement-map.

Florczyk, A. J., Ferri, S., Syrris, V., Kemper, T., Halkia, M., Soille, P., Pesaresi, M., 2016. A New European Settlement Map From Optical Remotely Sensed Data. In: IEEE Journal of Selected Topics in Applied Earth Observations and Remote Sensing 9 (5), 1978-1992,

https://dx.doi.org/10.1109/JSTARS.2015.2485662.

Hartmann, A., Meinel, G., Hecht, R., and Behnisch, M., 2016. A workflow for automatic quantification of structure and dynamic of the German building stock using official spatial data. In: ISPRS International Journal of Geo-Information 5 (2016) 8, 142, pp. 1-30, https://dx.doi.org/10.3390/ijgi5080142.

Jehling, M., Hecht, R., and Herold, H., 2016. Assessing urban containment policies within a suburban context - An approach to enable a regional perspective. Land Use Policy. Elsevier, Amsterdam https://doi.org/10.1016/j.landusepol.2016.10.031.

Kleemann, F., Lederer, J., Rechberger, H., and Fellner J., 2017. GIS-based Analysis of Vienna's Material Stock in Buildings. In: Journal of Industrial Ecology 21(2), pp. 368-380, https://dx.doi.org/10.1111/jiec.12446.

Schorcht, M., Krüger, T., and Meinel, G., 2016. Measuring Land Take: Usability of National Topographic Databases as Input for Land Use Change Analysis: A Case Study from Germany. In: ISPRS International Journal of Geo-Information 5 (2016) 8, 134, pp. 1-20, https://doi.org/10.3390/ijgi5080134. 